\title{
Bachelard, philosophe du jour, philosophe de la nuit
}

\author{
Véronique Le Ru
}

L'œuvre de Bachelard se partage en une philosophie des sciences qui a fait date par son engagement rationaliste, et en une métaphysique de l'imagination (Bachelard 1943 : 24), expression qu'utilise Bachelard lui-même dans L'Air et les songes pour désigner le but avoué de l'ensemble des textes de poétique qu'il a produits. Notre propos est de mettre en évidence le caractère double de son œuvre, non pas dans une visée simplement descriptive, mais véritablement problématique : nous voudrions montrer que cette caractéristique s'explique par le principe du double qui travaille la réflexion de Bachelard à la racine à la fois dans son œuvre épistémologique et dans son œuvre de poétique. Pour accomplir ce dessein, nous commencerons par donner quelques éléments de la biobibliographie de Bachelard et situerons sa réflexion dans le contexte philosophique et scientifique de l'époque. Nous approfondirons ensuite sa philosophie des sciences en montrant qu'elle se dédouble elle aussi en une prise de parti pour la science et un refus de prendre parti en philosophie qui le conduit peut-être à une certaine illusion épistémologique qu'il nous faudra préciser. Nous aborderons ensuite sa métaphysique de l'imagination qui se dédouble également, d'une part, en des considérations poétiques sur les quatre éléments, sur l'instant, sur l'espace, sur le rythme dont nous livrerons quelques exemples et, d'autre part, en des analyses sensibles sur la poésie et sur les poètes. Enfin nous réfléchirons à la manière dont Bachelard se pense lui-même et pense son œuvre : est-ce dans le miroir qu'il se tend à lui-même qu'on peut saisir le principe du double en acte dans un instantané autobiographique ?

\section{Quelques éléments de la vie et de l'œuvre de Bachelard}

Bachelard est né le 27 juin 1884 à Bar-sur-Aube et meurt à Paris le 16 octobre 1962. Il est d'abord employé des postes puis professeur de physique et de chimie au collège de Bar-surAube avant de passer tardivement en 1922, à l'âge de 38 ans, l'agrégation de philosophie. En 1928, il publie sa thèse : Essai sur une connaissance approchée, en même temps que sa thèse complémentaire, Étude sur l'évolution d'un problème de physique : la propagation thermique dans les solides, l'une et l'autre chez Vrin. Nous retiendrons de sa thèse les termes de « connaissance approchée » qui, déjà, expriment le style de Bachelard : la science tout entière est une connaissance approchée, qui a sa réalité et sa légitimité dans l'approche des phénomènes toujours à rectifier, toujours à affiner, toujours à construire. Qualifier la science de connaissance approchée ne signifie aucunement la rendre floue et incertaine ni l'enserrer dans un relativisme culturel mais au contraire la désigner comme un processus d'approche et d'invention du vrai, ce qui suppose des ruptures avec des conceptions qui s'avèrent erronées et périmées, et ce qui fait dire à Bachelard que « cette perspective d'erreurs rectifiées [...] caractérise [...] la pensée scientifique $\gg(\text { Bachelard } 1986: 10)^{1}$

À partir de la parution de sa thèse, Bachelard publie régulièrement des ouvrages de philosophie des sciences : un livre tous les deux ans en moyenne jusqu'en 1953 où paraît Le Matérialisme rationnel, son dernier ouvrage d'épistémologie. Pourtant sa bibliographie ne s'arrête pas là car verront encore le jour de son vivant quatre ouvrages de poétique : en 1957 , Châteaux en Espagne, la philosophie d'un graveur (Albert Flocon), en 1957 toujours, La Poétique de l'espace, en 1960, La Poétique de la rêverie et, en 1961, La Flamme d'une

\footnotetext{
1 Dans le chapitre conclusif de son ouvrage initialement paru en 1938, il reprend cette idée que l'esprit scientifique se constitue « comme un ensemble d'erreurs rectifiées » (Bachelard 1986 : 239).
} 
chandelle. Enfin, en 1970, sera publié de manière posthume un recueil de textes intitulé Le Droit de rêver. La publication de son œuvre de poétique commence en 1932, année féconde puisqu'il publie à la fois un ouvrage d'épistémologie, Le Pluralisme cohérent de la chimie moderne, et un ouvrage de poétique, L'intuition de l'instant. Six ans plus tard, survient une autre année doublement féconde avec La formation de l'esprit scientifique, grand texte d'épistémologie où il élabore la notion centrale d'obstacle épistémologique, et le premier ouvrage qu'il consacre aux quatre éléments de poétique : La Psychanalyse du feu. Suivront L'Eau et les rêves en 1942, L'Air et les songes en 1943, et en 1948 les deux ouvrages sur la Terre : La Terre et les rêveries de la volonté et La Terre et les rêveries du repos. La publication de l'œuvre de poétique commence donc en 1932 et s'achève en 1961 ou 1970, si l'on prend en compte l'essai posthume du Droit de rêver. De ce versant de l'œuvre, nous retiendrons précisément le droit de rêver, la rêverie (ce terme apparaît dans un nombre considérable de titres), comme douce pérégrination dans l'imaginaire.

Si l'on considère le corpus de Bachelard, on est frappé par l'équilibre : une douzaine d'ouvrages d'épistémologie, une douzaine d'ouvrages de poétique. Certaines années particulièrement fécondes, Bachelard fait coup double : en 1932 il publie, nous l'avons vu, Le Pluralisme cohérent de la chimie moderne et L'intuition de l'instant ; en 1938 La Formation de l'esprit scientifique et La Psychanalyse du feu; en 1940 Lautréamont et La Philosophie du non. Et il est tout à fait intéressant de noter qu'il travaille sur les mêmes objets dans les deux versants de son œuvre : l'espace, le temps (la durée, l'instant), la matière physico-chimique ou les éléments. Principe du double à l'œuvre dans la bibliographie, doublé d'un principe du double à l'œuvre dans ses enquêtes : les mêmes objets sont soumis à l'enquête épistémologique ou poétique. Tout cela ne peut être le fruit du hasard et il nous faut résoudre l'énigme de cette œuvre duelle. Mais encore un mot sur la biographie.

Bachelard est nommé en 1940 Professeur à la Sorbonne à la chaire d'histoire et de philosophie des sciences créée en 1909. Cette création est d'une grande nouveauté dans la philosophie française du début du siècle tout empreinte de spiritualisme et qui ne se préoccupe guère de la science actuelle ni de culture scientifique au-delà de Newton. Ravaisson, Maine de Biran, Blondel, Lavelle, Tarde, autant de noms illustres à l'époque qui confirment la teinture officielle qu'a la philosophie depuis plus d'un siècle: le spiritualisme. Quel est-il ? Cette philosophie en deux mots a pour objet de célébrer les valeurs morales de la liberté humaine et surtout de la liberté de l'esprit humain. L'esprit humain est en effet le thème de prédilection du spiritualisme en ce qu'il permet de décliner toutes les perspectives scientifique, esthétique, morale ou religieuse en un même hymne à la création spirituelle. La célébration des valeurs morales autour de l'esprit humain conduit à lier toutes les doctrines philosophiques de Platon à Kant en passant par Descartes et une métaphysique idéaliste : l'édifice de la philosophie française au début du $\mathrm{xx}^{\mathrm{e}}$ siècle est tout entière une machine de guerre contre le matérialisme français des Lumières. Le spiritualisme est destiné à mener une lutte idéologique contre les Lumières, ce qui a deux conséquences qui marquent la philosophie française du début $\mathrm{du} \mathrm{xx}^{\mathrm{e}}$ siècle, à savoir un rejet du positivisme d'Auguste Comte, jugé trop proche de Condorcet et de cette même philosophie des Lumières, et une évolution interne vers une philosophie ouvertement religieuse.

Tel est le décor philosophique dans lequel Bachelard entre et au sein duquel il va produire beaucoup de remous du fait de la révolution qu'il opère dans sa réflexion sur les rapports entre la philosophie et la science d'une part, et dans les relations qu'il invente entre la philosophie et la poésie d'autre part. 


\section{Bachelard et sa refonte des rapports entre philosophie et science}

La Philosophie du non, titre qu'il donne à son ouvrage de 1940 n'est pas une philosophie de la négation mais plutôt «une philosophie qui veut être vraiment adéquate à la pensée scientifique en évolution constante » (Bachelard 1983 : 7). La philosophie du non est une philosophie au travail qui cherche à saisir les variations de sens d'un concept scientifique de manière à le généraliser par l'intégration de ses déterminations contradictoires, et le prendre pour modèle ou lui chercher un modèle, une forme. Bachelard caractérise en effet la pensée scientifique "par une énorme puissance d'intégration et une extrême liberté de variation » (Bachelard 1965 : 16). La philosophie du non n'est donc pas une philosophie de la négation mais une philosophie de la variation. Comme le remarque Canguilhem dans sa présentation de son maître Bachelard, "Dans la progression du savoir, le non n'a point le sens d'anti, la philosophie du non a été pensée sur le modèle des géométries non euclidiennes, sur le modèle des mécaniques non newtoniennes [...] Philosophie de la connaissance rectifiée, philosophie du fondement par récurrence, la dialectique selon Gaston Bachelard désigne comme un fait de culture le vecteur de l'approximation scientifique dont elle renforce le sens en le proposant comme règle : "En toute circonstances, l'immédiat doit céder le pas au construit" [Bachelard $1983:$ 144] » (Canguilhem 1983 : III, 207) ${ }^{2}$. Le terme dialectique que Bachelard affectionne pour caractériser le processus d'invention du vrai en science n'a rien à voir avec le sens hégélien de dépassement ou de sursomption (Aufhebung) d'un concept à partir du travail du négatif ; il n'a rien à voir non plus avec le sens marxiste de matérialisme dialectique qui vise à analyser les évolutions humaines à partir de déterminations socio-économiques, et encore moins avec le sens scolaire de la thèse, de l'antithèse et de la synthèse. Non, le terme de dialectique signifie le processus d'invention du vrai, il désigne la démarche même de la raison inventive, c'est-àdire de la science même. L'apport tout à fait nouveau de Bachelard en philosophie des sciences consiste à ne plus distinguer la raison comme ensemble de principes et la science qui serait le résultat de l'application de ces principes, ce qui plaçait la raison au-dessus de la science comme puissance de principes. Bachelard considère que «la science ordonne la philosophie » (Bachelard $1983: 22$ ), que c'est par l'invention et l'application des concepts qu'on trouve une forme à ceux-ci. Autrement dit, le principe vient à la fin mais, comme la science est en évolution constante, les principes aussi sont toujours en voie de constitution.

Bachelard opère ainsi une véritable révolution copernicienne: alors que traditionnellement, la philosophie est posée comme la reine des sciences, la science est désormais pensée comme ce qui donne du grain à moudre aux philosophes. Dans Le Nouvel esprit scientifique, il va même jusqu'à dire que « la science crée de la philosophie » (Bachelard 1971 : 3). Autrement dit, les philosophes doivent apprendre à entendre l'appel de sens de la science. Bachelard veut réveiller la philosophie de son sommeil dogmatique et susciter en elle le désir d'acquérir une culture scientifique à la hauteur de son temps. Car ce que Bachelard ne supporte pas, c'est l'arrogance des philosophes qui prétendent philosopher sans aucune culture scientifique ou, pire encore, qui prétendent dire ce qu'est la science alors que leur culture s'arrête à l'âge classique et à la solution kantienne qui donne un cadre à la mécanique newtonienne à partir de la géométrie euclidienne. Oui mais, depuis Kant, ont surgi les géométries non euclidiennes au XIX ${ }^{\mathrm{e}}$ siècle (Riemann, Lobatchevski) qui remettent en cause l'idée qu'il puisse y avoir une vérité absolue en mathématiques, et qui substituent à cette idée le fait qu'il existe plusieurs systèmes d'axiomes cohérents qui fondent diverses géométries euclidiennes et non euclidiennes. Oui mais, depuis Kant, la physique a subi deux tremblements

\footnotetext{
2 "III. Dialectique et philosophie du non chez Gaston Bachelard » est le dernier des trois articles du chapitre consacré à Bachelard dans Études d'histoire et de philosophie des sciences, les deux autres étant : "I. L'histoire des sciences dans l'œuvre épistémologique de Gaston Bachelard », " II. Gaston Bachelard et les philosophes ».
} 
de terre avec la théorie de la relativité générale d'Einstein qui montre qu'il n'y a ni temps absolu, ni espace absolu, ni référentiel absolu, ni simultanéité absolue (ce qui bouleverse toutes nos représentations de l'espace et du temps), et enfin avec la mécanique quantique et les relations d'indétermination d'Heisenberg qui montre qu'au niveau corpusculaire, on ne peut mesurer à la fois la position et la vitesse d'une particule car l'appareil de mesure (l'accélérateur de particules) déplace l'objet à mesurer de manière non négligeable: on ne peut donc qu'assigner un degré de probabilité de position et de vitesse à un nuage corpusculaire (ce qui bouleverse complètement notre représentation d'un objet).

Bachelard veut que la philosophie prenne acte de tous ces changements conceptuels et qu'elle arrête de se donner des cadres de pensée périmés. Comme le dit Canguilhem en parlant de ce qu'attend Bachelard du philosophe, «Le philosophe doit sortir de la caverne philosophique, s'il ne veut pas se condamner à se repaître d'ombres, cependant que les savants non seulement voient la lumière mais la font »(Canguilhem 1983 : II, 187). Mais il ne s'agit pas simplement que la philosophie se mette à jour dans ses connaissances scientifiques, il faut qu'elle se réforme en profondeur et comprenne que les concepts innovants, féconds, heuristiques ne viennent pas de son fonds de bibliothèque mais de la science. Bachelard luimême souligne que ce n'est pas dans telle ou telle philosophie qu'il recherche les lignes fortes de son épistémologie mais qu'il les cherche dans les mémoires et les traités scientifiques. Et quand il use de concepts venant de la philosophie, il le fait très librement, sans égard pour le sens propre que les psychanalystes donnent à la psychanalyse, les dialecticiens à la dialectique, ou les phénoménologues à la phénoménologie. Il utilise ces termes comme des outils qu'il met au service d'un «polyphilosophisme $»^{3}$ (Bachelard 1949b : 36) cherchant à être adéquat avec la science de son temps : une sorte d'éclectisme philosophique au service de la science en mouvement.

Bachelard prend donc ouvertement parti pour la science mais, en revanche, refuse de prendre parti en philosophie, ce qui le conduit à une certaine illusion épistémologique dans son exigence d'adéquation de la philosophie à la science. Car si la science ordonne la philosophie, si la science impose ses valeurs objectives, il faut ajouter que cela se fait «à la longue » et non pas instantanément, car cela passe par des individus qui eux-mêmes sont porteurs de valeurs morales, philosophiques, esthétiques, qui parfois se heurtent aux propres avancées scientifiques dont ils sont pourtant les vecteurs. On peut ici prendre l'exemple de Pascal qui, au XVII ${ }^{\mathrm{e}}$ siècle, avait réuni tous les éléments pour élaborer la théorie du calcul infinitésimal et qui pourtant n'a pas construit cette théorie, ce qui a conduit Leibniz (l'inventeur du calcul infinitésimal) à penser que Pascal avait les yeux bandés par la religion. Il y a donc un dialogue à instruire entre savants et philosophes et le savant qui prétend inventer tout seul la philosophie des sciences est aussi arrogant que le philosophe enfermé dans sa caverne, décrié par Bachelard. Le principe du double doit ici se mettre en branle sans doute de manière plus réciproque que ne l'a pensé Bachelard: de la science à la philosophie, certes la conséquence est bonne, mais de la philosophie à la science, la conséquence est bonne également : il ne faut pas seulement chercher l'adéquation de la philosophie à la science, mais aussi les interactions entre la philosophie et la science. Si l'on mobilise la notion centrale d'obstacle épistémologique forgée par Bachelard comme ce qui fait écran à la pensée, comme ce qui empêche la pensée de se construire (voir Bachelard 1986, chap. 1: 13-22), il faut bien reconnaître qu'une notion scientifique, par ses strates à la fois philosophiques et scientifiques, peut faire obstacle à la science elle-même, on peut penser ici à la notion de force qui a suscité des querelles très longues entre scientifiques avant d'être reconduite à un problème de notation et donc à une convention. De surcroît, il n'est pas sûr que toute la créativité, toute la puissance d'inventer soit du côté de la science et non de la philosophie : à vrai dire, l'invention d'un concept puise dans la poésie, dans l'art, dans la

\footnotetext{
${ }^{3}$ Le passage où le terme apparaît est le suivant : « Nous croyons à la nécessité pour une épistémologie complète d'adhérer à un polyphilosophisme » (ibid. : 36).
} 
philosophie et dans la science. Si Bachelard hérite de Socrate dans son désir de connaitre "Connaître ne peut éveiller qu'un seul désir: connaître davantage, connaître mieux » (Bachelard 1965 : 223) -, il a sans doute oublié trop vite que le désir de connaître est autant le partage du philosophe, de l'artiste, du poète que du savant puisqu'il se nourrit avant tout du dialogue. L'illusion épistémologique à laquelle cède Bachelard est de mettre la créativité uniquement du côté de la science et de réduire la philosophie au rôle de miroir d'une science en mouvement, alors que science et philosophie s'entretiennent au sens fort du terme, elles s'enrichissent l'une l'autre par le dialogue mutuel qu'elles instaurent ${ }^{4}$.

Si l'on aborde à présent le versant poétique de l'œuvre de Bachelard à partir de ce même principe du double, comment la philosophie et la poésie s'articulent-elles ?

\section{Bachelard : l'invention de relations entre poésie et philosophie}

Comme Bachelard le dit lui-même dans L'Air et les songes, les quatre éléments et les considérations de poètes ou de penseurs sur ces quatre éléments sont des prétextes pour développer ses propres thèses sur la métaphysique de l'imagination « qui reste partout notre but avoué » (Bachelard 1943 : 24). Mais qu'entend-il par métaphysique de l'imagination ? Il signifie d'abord que la quête autour de la poésie est métaphysique, il s'agit d'assigner une origine ou un fondement à l'acte poétique. Or cette origine, pour Bachelard, est à chercher dans l'enfance et, plus précisément, dans les rythmes de l'enfance : "L'enfance est la source de nos rythmes. C'est dans l'enfance que les rythmes sont fondateurs et créateurs » (Bachelard 1950 : 149). La métaphysique de l'imagination est une quête pour faire revivre en soi les rythmes de l'enfance. Quels meilleurs moyens pour cela que de se plonger dans la poésie qui retaille par les rythmes singuliers de la langue le tissu d'existence des rythmes de l'enfance, des premières émotions et des premières rêveries? Mais cette retaille du tissu de l'enfance demande du travail, de l'expérience, du voyage. La poésie n'est pas l'expression des premières perceptions, elle est interprétation par l'imagination des émotions qui surviennent de ces perceptions : "Par l'imagination nous abandonnons le cours ordinaire des choses. Percevoir et imaginer sont aussi antithétiques que présence et absence. Imaginer c'est s'absenter, c'est s'élancer vers une vie nouvelle » (Bachelard 1943 : 10). La poésie n'est pas non plus l'expression simple de la rêverie car dans la rêverie on se laisse aller, on n'est ni dans la tension ni dans l'invention : "La rêverie se contente de nous transporter ailleurs sans que nous puissions vraiment vivre toutes les images du parcours. Le rêveur s'en va à la dérive » (id., ibid.).

La poésie, comme dit Guillevic dans Carnac du recueil Terraqué (1942), c'est autre chose, la poésie est une langue dans la langue, le poète dit encore dans Choses parlées, entretiens (1982) que la poésie est le seul moyen d'aborder par les mots, quand on sait le faire, le son intérieur de tout réel. Rien d'étonnant à ce que Bachelard fréquente Guillevic dans sa quête de la métaphysique de l'imagination. Ainsi, dans son enquête sur la poétique du vent, il cite le recueil Terraqué de Guillevic (Bachelard 1943 : 262).

Un [autre] Breton, dans un poème admirablement limité au noyau poétique des impressions, écrit :

$$
\begin{aligned}
& \text { «Il y a quelqu'un } \\
& \text { Dans le vent ». }
\end{aligned}
$$

La poésie n'est ni la perception ni la rêverie mais s'en nourrit, elle en fait son miel, mais faire son miel est un travail, on appelle les abeilles de la ruche les ouvrières. Pour filer la métaphore, les abeilles butineuses viennent recueillir les rythmes de l'enfance, les rêveries, pour les déposer

\footnotetext{
${ }^{4}$ Sur l'illusion épistémologique de Bachelard, voir Lecourt (1974 :165-169).
} 
dans la ruche où les abeilles ouvrières prennent le relais pour en faire le miel. Ce travail est long et difficile car, comme le remarque Bachelard (ibid. : 12) :

Il faut [...] appeler l'objet poétique par son nom, par son vieux nom, en lui donnant son juste nombre sonore, en l'entourant des résonateurs qu'il va faire parler, des adjectifs qui vont prolonger sa cadence, sa vie temporelle.

Et Bachelard poursuit en citant Les cahiers de Malte Laurids Bridge de Rilke (id., ibid.) :

Pour écrire un seul vers, il faut avoir vu beaucoup de villes, d'hommes et de choses, il faut connaître les animaux, il faut sentir comment volent les oiseaux et savoir quel mouvement font les petites fleurs en s'ouvrant le matin.

Mais le plus étonnant, dans la suite du texte, est que Bachelard lui-même se met à la place du poète (id., ibid.) :

Chaque objet contemplé, chaque grand nom murmuré est le départ d'un rêve et d'un vers, c'est un mouvement linguistique créateur. Que de fois au bord du puits, sur la vieille pierre couverte d'oseille sauvage et de fougère, j'ai murmuré le nom des eaux lointaines, le nom du monde enseveli...Que de fois l'univers m'a soudain répondu... Ô mes objets ! comme nous avons parlé !

L'aveu profond de communion poétique avec l'univers se combine ici à un aveu de vocation ratée : Bachelard ne se reconnait-il pas poète ? Et ne peut-on pas alors lire son œuvre de poétique comme celle d'un poète contrarié ; de la même façon, ne peut-on pas appréhender son œuvre de philosophe des sciences comme celle d'un savant contrarié ?

En tout cas, on peut y voir à l'œuvre le principe du double. De même que Bachelard étudie au plus près la science au travail afin de construire une philosophie de la science au travail, de même Bachelard étudie au plus près l'imagination au travail dans la poésie afin de construire une métaphysique de l'imagination. Mais peut-être y a-t-il aussi comme un regret dans l'œuvre double de Bachelard, peut-être y a-t-il comme un aveu de double vocation contrariée car Bachelard sait qu'il n'est ni un savant ni un poète et que son œuvre ne peut s'inscrire que comme une doublure de la poésie ou de la science : la philosophie comme une doublure adéquate de la science, la métaphysique de l'imagination comme une doublure adéquate de la poésie.

En ce sens, notre proposition de lecture de son œuvre autour du principe du double s'inscrit dans le sillage de Michel Fichant (1975: 100-114) qui cherche à penser dans l'unité de discours ou encore dans l'unité du style, l'unité de l'œuvre de Bachelard. Beaucoup de commentateurs se sont cassé les dents sur cette question de l'unité des écrits de Bachelard et celui-ci n'a cessé de dire que son œuvre de poétique était irréductible à son œuvre d'épistémologue car "images et concepts se forment à ces deux pôles opposés de l'activité psychique que sont l'imagination et la raison. Joue entre elles une polarité d'exclusion. Ici les pôles opposés ne s'attirent pas ; ils se repoussent » (Bachelard 1960 : 47). Et il ajoute (id., ibid.) :

Je l'ai compris trop tard. Trop tard, j'ai connu la bonne conscience dans le travail alterné des images et des concepts, deux bonnes consciences qui seraient celle du plein jour, et celle qui accepte le côté nocturne de l'âme. Pour que je jouisse de double bonne conscience, la bonne conscience de ma double nature enfin reconnue, il faudrait que je puisse faire encore deux livres : un livre sur le rationalisme appliqué, un livre sur l'imagination active. Une bonne conscience, c'est pour moi, si insuffisantes que soient les œuvres, une conscience occupée - jamais vide - la conscience d'un homme au travail jusqu'à son dernier souffle.

Bachelard écrit ces lignes en 1960 ; il a alors 76 ans et ce qui est étrange est qu'il a publié en 1949 Le Rationalisme appliqué et en 1953 son dernier livre d'épistémologie Le Matérialisme rationnel - il n'en publiera pas d'autre. Cela veut dire que le rationalisme appliqué pour lui ne 
désigne pas le titre d'un livre mais une méthode de travail : la philosophie au travail cherchant à être adéquate à la science au travail. Dans La Philosophie du Non, Bachelard déclare :

«Si l'on pouvait traduire philosophiquement le double mouvement qui anime la pensée scientifique, on s'apercevrait que $[\ldots]$ l'empirisme et le rationalisme sont liés $[\ldots]$ l'empirisme a besoin d'être compris ; le rationalisme a besoin d'être appliqué ».

(Bachelard $1983: 5-6)$

Depuis la publication de sa thèse en 1928, se sont écoulés trente-deux ans de publication continue autour du feu, de la science, de la poésie, et pourtant Bachelard exprime cette insatisfaction foncière de la conscience qui ne peut être bonne sans avoir encore écrit ces livres, sans avoir travaillé encore sur le rationalisme appliqué et sur l'imagination active. Si Bachelard a fait de la rêverie et du repos deux thèmes de prédilection dans ses recherches, il ne s'est guère reposé, ce qui le caractérise, c'est plutôt sa force de travail, l'inquiétude de conscience qui le reconduit toujours à sa table de travail : « la pensée anxieuse » (Bachelard 1971 : 177) dont il parle dans Le Nouvel esprit scientifique, ou « cette raison risquée, sans cesse reformée, toujours auto-polémique » (Bachelard $1949: 47)$ est bien la sienne.

En 1960, quand il fait part de ses morsures de conscience dans La Poétique de la rêverie, il reste à Bachelard deux ans à vivre mais dans ces deux ans il publiera encore en 1961 un dernier livre sur l'imagination vive : La Flamme d'une chandelle. Son premier texte sur les éléments de la matière, La psychanalyse du feu, il l'avait publiée en 1938, à l'âge de 54 ans ; sa dernière œuvre de poétique, La Flamme d'une chandelle, il la publie en 1961, à l'âge de 77 ans. Le feu est devenu flamme, mais quelle flamme ! La Flamme d'une chandelle apparaît à bien des égards comme un testament philosophique et comme un autoportrait. Ainsi Bachelard écrit $(1961: 3)$ :

Tout rêveur de flamme est un poète en puissance. Toute rêverie devant la flamme est une rêverie qui admire $[\ldots]$ Cette admiration première est enracinée dans notre lointain passé [...] La flamme nous appelle à voir en première fois : nous en avons mille souvenirs, [...] le rêveur vit dans un passé qui n'est plus uniquement le sien, dans le passé des premiers feux du monde.

\section{Conclusion : l'unité de l'œuvre}

Il est temps, après ce parcours dans son œuvre de philosophie des sciences et de poétique de revenir sur le titre que nous avons choisi : Bachelard, philosophe du jour, philosophe de la nuit. Dans l'aveu de La Poétique de la rêverie, il se définit lui-même dans sa double nature marquée par l'attrait des images et des concepts et par le travail alterné des images et des concepts : « deux bonnes consciences qui seraient celle du plein jour, et celle qui accepte le côté nocturne de l'âme » (Bachelard 1960 : 47). Or ce qui est intéressant est que le jour et la nuit, le matin et le soir marquent tout processus d'invention : le savant aussi a une double vie et une double conscience que Bachelard sollicite dans La philosophie du non (1983 : 13) :

\footnotetext{
Dites-nous ce que vous pensez, non pas en sortant du laboratoire, mais aux heures où vous quittez la vie commune pour entrer dans la vie scientifique. Donnez-nous, non pas votre empirisme du soir, mais votre vigoureux rationalisme du matin, l'a priori de votre rêverie mathématique, la fougue de vos projets, vos intuitions inavouées.
}

Ce qui intéresse fondamentalement Bachelard dans la science au travail, ce sont les tâtonnements, les essais, les erreurs : « Nous demanderons donc aux savants : comment pensezvous, quels sont vos tâtonnements, vos essais, vos erreurs ?» (id., ibid.). Comme le souligne Canguilhem, Bachelard a saisi de manière lumineuse l'inhérence du trouble et de l'erreur à la science (Canguilhem $1983: 204)$ : 
Descartes montrait comment l'erreur est possible. Bachelard la montre nécessaire, non par le fait de ce qui est extérieur à la connaissance mais par l'acte même de connaissance. 'C'est dans l'acte même de connaître, intimement, qu'apparaissent par une sorte de nécessité fonctionnelle des lenteurs et des troubles". [Bachelard $1986: 13]$ )

Selon Canguilhem, Bachelard, par son concept d'obstacle épistémologique, a fondé positivement l'obligation d'errer. Or à cette obligation d'errer pour le philosophe du jour qu'est Bachelard, répond le droit de rêver pour le philosophe de la nuit, qu'il est également. Dans $L a$ Terre et les rêveries de la volonté, il se donne comme tâche de « rêver la matière » (Bachelard 1948 : 169). On a vu que dans les deux versants de son œuvre, il se penche sur les mêmes objets : la matière, ses quatre éléments, l'espace, la durée, l'instant. La matière est bien ce qui est travaillé par le principe du double qui caractérise la philosophie de Bachelard : la matière est façonnée par un rationalisme appliqué mais aussi par l'imagination au travail. Rêver la matière pour mieux l'imaginer dans la nuit et pour mieux la connaître dans le jour. Ici se noue l'unité de l'œuvre dans cette tâche toujours recommencée de rêver la matière : "On ne peut étudier que ce qu'on a d'abord rêvé. La science se forme plutôt sur une rêverie que sur une expérience » (Bachelard 1949a: 48). Et l'ouvrage toujours remis sur le métier conduit et reconduit Bachelard à sa table d'existence en quête d'un double dans son œuvre (1961: 111) :

Oui, c'est à ma table d'existence que j'ai connu l'existence maxima [oui c'est bien cela], l'existence en tension [...] Tout autour de moi est repos, est tranquillité ; mon être seul, mon être qui cherche de l'être est tendu dans l'invraisemblable besoin d'être un autre être, un plus qu'être.

Toujours le principe du double à l'œuvre dans l'être même, toujours le principe du double à la racine de l'œuvre, que l'on peut lire par un jeu de miroir, dans le droit de rêver ou dans l'obligation d'errer puisque le double s'inscrit dans le verbe rêver qu'on peut déchiffrer comme un palindrome « re-v-er » et qu'il figure aussi structurellement dans le verbe errer « er-r-er ». 